\title{
ANALYSIS OF THE LONGITUDINAL COMPONENT OF THE ELECTRIC FIELD GENERATED BY FLAT AND PIXELATED LIQUID CRYSTAL DISPLAYS
}

\author{
Onur Kulce, Levent Onural
}

\author{
Department of Electrical and Electronics Engineering \\ Bilkent University, TR-06800 Bilkent, Ankara, Turkey
}

\begin{abstract}
The longitudinal, $z$, component of the electric field is investigated for the pixelated and flat liquid crystal displays (LCDs) for monochromatic case. The pixelation process is assumed to occur in free space. The $z$ component is computed in the Fourier domain by using Gauss's Law from the $x$ and $y$ components of the output electric field. The effect of the display parameters are discussed for a $y$ polarized display in a phase only operation. It is found that, in the low frequency regions, the size of the region of the large magnitudes becomes smaller as the width of the active region increases. Moreover, the validity of the scalar theory for a single pixel is evaluated for varying pixel sizes. It is shown that, when the ratio of the width of the active region to wavelength is between 1.5 and 5 , the error decays with oscillations between $43 \%$ and $5 \%$. When that ratio is larger than 15 , the error does not exceed $3 \%$.
\end{abstract}

Index Terms - liquid crystal displays, longitudinal component, validity of the scalar theory

\section{INTRODUCTION}

The liquid crystal displays (LCDs) are widely used both in commercial devices and research related purposes including 3DTV applications. Amplitude only and phase only spatial light modulators (SLMs) can be counted among those devices.

In the literature, the electromagnetic wave propagation in a LC medium in the in-line illumination case is usually treated as the propagation of an infinite extent plane wave which only has transverse field components $[1,2,3]$. Then, as a consequence of diffraction, plane wave components with oblique propagation angles arise and propagate in free space [2,3]. Furthermore, such diffraction effects are desirable for a display with large viewing angle. As the pixel size decreases, the relative power in larger angles increases, as well [4]. On the other hand, due to the transversality condition of the plane wave components, the longitudinal, $z$, component of the electric field exists at the oblique angles [5]. Since the $z$ component can be seen as the cause of error in the scalar approximation to the optical field, a trade-off between the use of the scalar theory and having large diffraction angles emerges.

In this paper, we compute the $z$ component of the monochromatic electric field produced by a flat panel pixelated LCD on the display surface and make a quantitative analysis on the error of using the scalar theory in such a display. We assume that the $x$ and $y$ components are generated as desired by the display within possible technical limitations as imposed by various types of LCDs. We also formulate the pixelation process as the multiplication of the $x$ and $y$ components by a regular rectangular grid and use Gauss's Law to find the $z$ component.

\section{PIXELATED TRANSVERSE FIELD IN THE SPACE DOMAIN AND ITS FOURIER TRANSFORM}

Since we assume that the field is monochromatic, the time dependence of the field is omitted. We also assume that the pixel configuration is regular rectangular and the phase and amplitude variations on the surface of the active region of a pixel are constant. At the $z=0$ plane, we write the continuous $x$ and $y$ components of the electric field generated by a single pixel as

$$
\hat{E}_{x, y}[n, m] \operatorname{rect}\left(\frac{x-n P}{\Delta}\right) \operatorname{rect}\left(\frac{y-m P}{\Delta}\right),
$$

where $\hat{E}_{x, y}[n, m] \in \mathbb{C}$ denote either the $x$ or $y$ component of the electric field produced at the pixel whose location is determined by the integers $n$ and $m, P \in \mathbb{R}^{+}$is the pixel period, $\Delta \in(0, P)$ is the width of the active region of the pixel and

$$
\operatorname{rect}(r)=\left\{\begin{array}{ll}
1 & \text { if }|r| \leq \frac{1}{2} \\
0 & \text { otherwise }
\end{array},\right.
$$

for $r \in \mathbb{R}$. The ratio $A_{r}=\Delta / P$ is called the fill factor or the aperture ratio.

Then, the sum of the $x$ and $y$ components of the field produced at the LCD exit can be written as

$$
\begin{aligned}
& E_{x, y}(x, y)=\sum_{n=0}^{N-1} \sum_{m=0}^{M-1} \hat{E}_{x, y}[n, m] \times \\
& \operatorname{rect}\left(\frac{x-n P}{\Delta}\right) \operatorname{rect}\left(\frac{y-m P}{\Delta}\right),
\end{aligned}
$$

where $N$ and $M$ are the number of columns and rows of the display, respectively and so, the display size is $M \times N$ pixels. We assume that the top-left corner pixel of the display corresponds to pixel index $n=m=0$.

Here we use the following definition of the two dimensional (2D) Fourier transform (FT) of a 2D function $f(x, y)$ :

$$
\mathcal{F}\left(k_{x}, k_{y}\right)=\iint_{-\infty}^{\infty} f(x, y) e^{-j\left(k_{x} x+k_{y} y\right)} \mathrm{d} x \mathrm{~d} y
$$

where $k_{x}, k_{y} \in \mathbb{R}$ are the frequency variables with the units of radians per unit length and $j=\sqrt{-1}$. Then, the 2D FTs of the $x$ and $y$ components at the $z=0$ plane that are given in Equation 3 become

$$
\mathcal{E}_{x, y}\left(k_{x}, k_{y}\right)=\frac{4 \sin \left(\frac{k_{x} \Delta}{2}\right) \sin \left(\frac{k_{y} \Delta}{2}\right)}{k_{x} k_{y}} \mathcal{D}_{x, y}^{P, N, M}\left(k_{x}, k_{y}\right)
$$


where

$$
\mathcal{D}_{x, y}^{P, N, M}\left(k_{x}, k_{y}\right)=\sum_{n=0}^{N-1} \sum_{m=0}^{M-1} \hat{E}_{x, y}[n, m] e^{-j P\left(k_{x} n+k_{y} m\right)} .
$$

Please note that, the only parameter of the display that does not have an effect on $\left.\mathcal{D}_{x, y}^{P, N}, k_{x}, k_{y}\right)$ is the width of the active region, $\Delta$. It can be verified that $\mathcal{D}_{x, y}^{P, N, M}\left(k_{x}, k_{y}\right)$ are periodic by $2 \pi / P$ radians per unit length along both $k_{x}$ and $k_{y}$ directions.

\section{FOURIER TRANSFORM OF THE $Z$ COMPONENT OF THE PIXELATED FIELD}

We assume that the pixelation process occurs in free space, which is an isotropic medium. With this assumption, the 2D FT of the $z$ component, $\mathcal{E}_{z}\left(k_{x}, k_{y}\right)$, over the display surface can be found using Gauss's Law as [5]:

$$
\mathcal{E}_{z}\left(k_{x}, k_{y}\right)=\frac{-k_{x} \mathcal{E}_{x}\left(k_{x}, k_{y}\right)-k_{y} \mathcal{E}_{y}\left(k_{x}, k_{y}\right)}{k_{z}}
$$

Since we assume that the field is monochromatic and we choose the $z$ component of the propagation (and decaying in the case of evanescent waves) direction is always positive for each plane wave component, $k_{z}$ equals to $\sqrt{k^{2}-k_{x}^{2}-k_{y}^{2}}$, where $k$ is the wave number and equals to $2 \pi / \lambda$, where $\lambda$ is the wavelength of the monochromatic field. We also assume that, $\mathcal{E}_{x}\left(k_{x}, k_{y}\right)=$ $\mathcal{E}_{y}\left(k_{x}, k_{y}\right)=\mathcal{E}_{z}\left(k_{x}, k_{y}\right)=0$ for $k_{z}=0$. This assumption leads to the restriction that $\mathcal{D}_{x, y}^{P, N, M}\left(k_{x}, k_{y}\right)$ are 0 when $\sqrt{k_{x}^{2}+k_{y}^{2}}$ $=2 \pi / \lambda$.

If we insert Equation 5 into Equation 7, we find

$$
\begin{gathered}
\mathcal{E}_{z}\left(k_{x}, k_{y}\right)=-\frac{\Delta^{2}}{k_{z}} \operatorname{sinc}\left(\frac{k_{x} \Delta}{2 \pi}\right) \operatorname{sinc}\left(\frac{k_{y} \Delta}{2 \pi}\right) \times \\
\left(k_{x} \mathcal{D}_{x}^{P, N, M}\left(k_{x}, k_{y}\right)+k_{y} \mathcal{D}_{y}^{P, N, M}\left(k_{x}, k_{y}\right)\right),
\end{gathered}
$$

where

$$
\operatorname{sinc}(r)=\frac{\sin (\pi r)}{\pi r} \text { for } r \in \mathbb{R}
$$

The 2D FT of the $z$ component of the electric field over all types of flat LCD surfaces can be computed using Equation 8 . Different configurations may impose some restrictions on the $x$ and $y$ components, $\hat{E}_{x, y}[n, m]$. For example, a phase only SLM may require $\hat{E}_{x}[n, m]=0$ and $\left|\hat{E}_{y}[n, m]\right|=C$ for all $n$ and $m$, where $C$ is a constant $[6,7]$. Or a twisted nematic (TN) LCD without analyzing polarizer requires $\left|\hat{E}_{x}[n, m]\right|^{2}+\left|\hat{E}_{y}[n, m]\right|^{2}=C$, where $C$ is again constant [1]. Moreover, in a TN-LCD without the analyzing polarizer, as a direct consequence of the linear polarization, the phases of the $x$ and $y$ components at each pixel are either equal or $\pi$ radians apart depending on the LC configuration.

\section{EFFECT OF THE DISPLAY PARAMETERS ON THE MAGNITUDE OF THE $Z$ COMPONENT}

Here, we investigate the effect of the display parameters on the magnitude of the $z$ component at different spatial frequencies. Since in some of the display applications, the field is assumed to be linearly polarized, in this section, we assume that the $x$ component of the field is zero; so, $\hat{E}_{x}[n, m]=0$ for all $n$ and $m$. In this case, the magnitude of $\mathcal{E}_{z}\left(k_{x}, k_{y}\right)$ given by Equation 8 becomes

$\left|\mathcal{E}_{z}\left(k_{x}, k_{y}\right)\right|=\left|\frac{2 \Delta}{k_{z}} \operatorname{sinc}\left(\frac{k_{x} \Delta}{2 \pi}\right) \sin \left(\frac{k_{y} \Delta}{2}\right) \mathcal{D}_{y}^{P, N, M}\left(k_{x}, k_{y}\right)\right|$
Firstly, it can be verified that, the rate of change of $1 / k_{z}$ with respect to $k_{x}$ and $k_{y}$ is much larger than the rate of change of $\operatorname{sinc}\left(\frac{k_{x} \Delta}{2 \pi}\right) \sin \left(\frac{k_{y} \Delta}{2}\right)$ term in the high frequency regions and much smaller in the low frequency regions. Therefore, the variation in the result of Equation 10 for different $k_{x}$ and $k_{y}$ values is mainly caused by $\mathcal{D}_{y} / k_{z}$ and $\mathcal{D}_{y} \operatorname{sinc}\left(\frac{k_{x} \Delta}{2 \pi}\right) \sin \left(\frac{k_{y} \Delta}{2}\right)$ in the high frequency and low frequency regions, respectively, where $\mathcal{D}_{y}$ is short for $\mathcal{D}_{y}^{P, N, M}\left(k_{x}, k_{y}\right)$. Moreover, if $P$ and $\Delta$ values are comparable and the rate of change of $\mathcal{D}_{y}$ is much smaller than the rate of change of $\operatorname{sinc}\left(\frac{k_{x} \Delta}{2 \pi}\right)$, due to the decaying characteristics of the sinc term, the periodic structure of $\mathcal{D}_{y}$ along the $k_{x}$ direction may not be seen in $\left|\mathcal{E}_{z}\left(k_{x}, k_{y}\right)\right|$ in the low frequency regions. As opposed to this, if the rate of change of $\mathcal{D}_{y}$ is much larger than the rate of change of the sinc term, then, the sinc pattern may not be seen in the low frequency regions. The rate of change relation between $\mathcal{D}_{y}^{P, N, M}$ and $1 / k_{z}$ is also similar in the high frequency regions.

In Figure 1, we present a simulation of Equation 10. In the simulation we assume that $\hat{E}_{y}[n, m]$ is $e^{j \phi[n, m]}$, where $\phi[n, m]$ is generated randomly for each $n$ and $m$ from an independent and uniform distribution between 0 and $2 \pi$ with 8 -bit precision. The reason of this choice is to excite the system at all frequency locations, as the power spectral density of the generated $\hat{E}_{y}[n, m]$ is flat. We also choose $N=1920$ and $M=1080$. In summary, our analysis covers a $y$ polarized display with a size of $1080 \times 1920$ pixels in a phase-only operation. Then, by using the generated $\hat{E}_{y}[n, m]$ and assuming $\lambda=500 \mathrm{~nm}$, we compute $\left|\mathcal{D}_{y}^{P, N, M}\left(k_{x}, k_{y}\right)\right|$ in a $512 \times 512$ frequency grid for $k_{x}, k_{y} \in$ $[-k, k)$ and for two pixel period values, $P=8 \mu \mathrm{m}$ and $P=20$ $\mu \mathrm{m}$. Note that, since we assume that the pixel count is always the same, different pixel periods yield different display sizes in terms of width and height. Finally, we compute three different $\mathcal{E}_{z}\left(k_{x}, k_{y}\right)$ s using Equation 10 for the pairs $(P, \Delta)=(8,7)$, $(8,2)$ and $(20,2)$, where all the units are in $\mu \mathrm{m}$.

In Figures $1 \mathrm{a}, 1 \mathrm{~b}$ and $1 \mathrm{c}$, the computed $\left|\mathcal{E}_{z}\left(k_{x}, k_{y}\right)\right| \mathrm{s}$ are shown in $\log$ scale for better illustration. Since $\mathcal{E}_{z}\left(k_{x}, k_{y}\right)$ can also be seen as the multiplication of $\mathcal{E}_{y}\left(k_{x}, k_{y}\right)$, which is computed using Equation 5 , and $-k_{y} / k_{z}$, some higher order lobes along the vertical direction appears in $\left|\mathcal{E}_{z}\left(k_{x}, k_{y}\right)\right|$ pattern. Please note that, although it is not shown here, it can be said that these higher order lobes are not so strong in $\left|\mathcal{E}_{y}\left(k_{x}, k_{y}\right)\right|$ pattern. The width of these lobes along both the horizontal and vertical directions mainly depend on $\Delta$ rather than $P$. Also the size of the lobes gets larger as $\Delta$ gets smaller. In all three cases, in most of the high frequency regions, the magnitude of the $z$ component increases sharply by virtue of the $1 / k_{z}$ multiplier. Also, although it is not clear from the figures due to the log scale illustration, these high frequency regions are the locations with the largest magnitude by far. Finally, if the display was $x$ polarized instead of a $y$ polarized one, then, the spectrums of the $z$ component would be 90 degree rotated versions of the ones shown in Figure 1 since the $x$ component would be multiplied by $-k_{x} / k_{z}$ instead of $-k_{y} / k_{z}$.

In $(P, \Delta)=(20,2)$ case, since $P$ is much larger than $\Delta$, it is possible to observe an approximate periodic structure in the low frequency region along both $k_{x}$ and $k_{y}$ directions. In this respect, in Figures $2 \mathrm{a}$ and $2 \mathrm{~b}$, we present $1 \mathrm{D}$ horizontal and vertical cross sections of $\mathcal{E}_{z}\left(k_{x}, k_{y}\right)$ for $k_{y}=k / 8$ and $k_{x}=0$, respectively. It can be seen from Figure 2a that, in the low frequency region, only two periods of $\left|\mathcal{D}_{y}^{P, N, M}\left(k_{x}, k_{y}\right)\right|$ do not vanish and the rest of the signal decays due to the sinc term. In Figure $2 b$, on the other hand, due to the $\sin$ term, $\left|\mathcal{E}_{z}\left(k_{x}, k_{y}\right)\right|$ shows a quasi-periodic structure and increases in the high frequency regions due to the $1 / k_{z}$ term. 


\section{VALIDITY OF THE SCALAR APPROXIMATION IN THE DISPLAYS}

In the amplitude and phase only applications, the light field is generally treated as a scalar quantity. If the output field is linearly polarized, the scalar field can be assumed as the field produced by that linearly polarized electric field. However, for intensity recording mediums, such as charged coupled device (CCD) cameras or the human eye, the $z$ component affects the recorded data and recorded intensity becomes the sum of the magnitude squares of the assumed scalar field and the $z$ component. Therefore, the $z$ component can be seen as the source of the error of the scalar theory when the intensity of the field is recorded.

In order to compute the amount of error in the scalar approximation, we define the ratio of the energy of the $z$ component to the total energy of the electric field at the $z=0$ plane as the error measure, $\epsilon$. This ratio can be written as

$\epsilon=\frac{\iint_{-\infty}^{\infty}\left|\mathcal{E}_{z}\left(k_{x}, k_{y}\right)\right|^{2} \mathrm{~d} k_{x} \mathrm{~d} k_{y}}{\iint_{-\infty}^{\infty}\left(\left|\mathcal{E}_{x}\left(k_{x}, k_{y}\right)\right|^{2}+\left|\mathcal{E}_{y}\left(k_{x}, k_{y}\right)\right|^{2}+\left|\mathcal{E}_{z}\left(k_{x}, k_{y}\right)\right|^{2}\right) \mathrm{d} k_{x} \mathrm{~d} k_{y}}$

For the linearly polarized case with zero phase difference between the $x$ and $y$ components, these components can be written as $\hat{E}_{x}[n, m]=c \hat{E}[n, m]$ and $\hat{E}_{y}[n, m]=s \hat{E}[n, m]$, respectively, where $c$ and $s$ are some nonnegative real numbers such that $c^{2}+$ $s^{2}=1$ and $\hat{E}[\cdot, \cdot] \in \mathbb{C}$. The values of $c$ and $s$ depend on the design configuration of the LCD. If, for example, the output field is $y$ polarized, then, $c=0, s=1$, or in the case of diagonally linearly polarized case, $c=s=1 / \sqrt{2}$. Then, for the propagating fields, Equation 11 becomes

$\epsilon=\frac{\iint_{P} \operatorname{sinc}^{2}\left(\frac{k_{x} \Delta}{2 \pi}\right) \operatorname{sinc}^{2}\left(\frac{k_{y} \Delta}{2 \pi}\right)|\mathcal{D}|^{2}\left(\frac{c k_{x}+s k_{y}}{k_{z}}\right)^{2} \mathrm{~d} k_{x} \mathrm{~d} k_{y}}{\iint_{P} \operatorname{sinc}^{2}\left(\frac{k_{x} \Delta}{2 \pi}\right) \operatorname{sinc}^{2}\left(\frac{k_{y} \Delta}{2 \pi}\right)|\mathcal{D}|^{2}\left[1+\left(\frac{c k_{x}+s k_{y}}{k_{z}}\right)^{2}\right] \mathrm{d} k_{x} \mathrm{~d} k_{y}}$

where, $\mathcal{D}$ is assumed to be computed using $\hat{E}[n, m]$ and a function of $k_{x}$ and $k_{y}$. Also, the subscript of the integral symbols, $P$, indicates that the integration is computed in the propagating region.

In a single pixel analysis, in which $|\mathcal{D}|$ becomes constant for all $k_{x}$ and $k_{y}$ values, it can be shown that, the result of Equation 12 is independent from $c$ and $s$ values. Moreover, the terms within the integrands can be written as a function of $\Delta / \lambda$. In Figure 3, we show the percent error on the scalar theory for a single pixel, $\epsilon$, as a function of $\Delta / \lambda$ which is assumed to vary between 1.5 and 25 for $\lambda=500 \mathrm{~nm}$. We compute the integrals in Equation 12 by using a numeric method. In the figure, although the error decreases as $\Delta / \lambda$ increases in general trend, this decrease is not monotonic and there are some local oscillations, especially for the small values of $\Delta / \lambda$. For example, when $1.5 \leq \Delta / \lambda \leq 5$, the error oscillates between $43 \%$ and $5 \%$. At these locations, smaller errors can be obtained even if $\Delta / \lambda$ decreases. For example, when $\Delta / \lambda$ is 2.5 , the introduced error is $27 \%$. However, when $\Delta / \lambda$ is 2 , the introduced error is $12 \%$. For the large values of $\Delta / \lambda$, on the other hand, there is almost no oscillation and when $\Delta / \lambda>15$, the error percentage is at most $3 \%$.

As opposed to the single pixel analysis, if $\left|\mathcal{D}\left(k_{x}, k_{y}\right)\right|$ shows an unequal distribution over the frequency domain, then the error on the scalar theory may depend on the values of $c$ and $s$. In order to show this, we consider two cases, that are $c=1, s=0$ and $c=0, s=1$. In these cases, in the computation of the energy of the $z$ component, as shown in Equation $12,\left|\mathcal{D}\left(k_{x}, k_{y}\right)\right|^{2}$ is multiplied by $k_{x}^{2}$ and $k_{y}^{2}$, respectively. If, for example, $\left|\mathcal{D}\left(k_{x}, k_{y}\right)\right|$ is large for the large values of $k_{x}$, then the error in the $c=1, s=0$ case becomes larger than the $c=0, s=1$ case. This means that, if the LCD configuration is such that the output is linearly polarized along the $x$ direction, then the scalar theory becomes more erroneous for the electric fields whose propagation direction along the $x$ direction is larger than the propagation direction along the $y$ direction. The inverse is also valid. Moreover, this fact can be generalized for arbitrary directions as follows: If the propagation direction of the scalar wave is dominant along a certain direction at the $x-y$ plane, then the scalar theory becomes more accurate if the direction of the linear polarization of the outgoing wave is vertical to the dominant direction of the scalar wave.

\section{CONCLUSIONS}

In this work, the $z$ component of a monochromatic electric field produced by a flat and pixelated LCD is analyzed. For a phase only $y$ polarized display, a simulation is presented in order to investigate the effect of different parameters of the display on the magnitude of the 2D Fourier transform of the $z$ component. In addition to these, the validity of the scalar approximation in the LCDs are quantitatively evaluated. We found that, for a single pixel, the validity of the scalar approximation decreases as the width of the active region of the pixel decreases as well. Moreover, when the output field is generated through multiple pixels, and if the propagation direction of plane wave components of an electric field are confined in a narrow band in $k_{x}-k_{y}$ plane, then, the error in the scalar approximation is minimized if the output linear polarization direction becomes vertical to the propagation direction of the plane waves.

\section{ACKNOWLEDGEMENTS}

Onur Kulce acknowledges the partial support of TÜBİTAK for this work in the form of a scholarship.

\section{REFERENCES}

[1] P. Yeh and C. Gu, Optics of Liquid Crystal Displays, Wiley, 2nd edition, 2009.

[2] E. A. B. Saleh and M. C. Teich, Fundamentals of Photonics, Wiley, 2nd edition, 2007.

[3] J. W. Goodman, Introduction to Fourier Optics, McGrawHill, 2nd edition, 1996.

[4] L. Onural, F. Yaras, and H. Kang, "Digital holographic threedimensional video displays," Proceedings of the IEEE, vol. 99, no. 4, pp. 576-589, April 2011.

[5] C. A. Balanis, Antenna Theory: Analysis and Design, Wiley, 3rd edition, Apr. 2005.

[6] Z. Zhang, Z. You, and D. Chu, "Fundamentals of phase-only liquid crystal on silicon (LCOS) devices," Light: Sci. \& Appl., vol. 3, no. 10, pp. e213, 2014.

[7] S. Osten, S. Krüger, and A. Steinhoff, "Spatial light modulators based on reflective micro-displays," Technisches Messen, vol. 73, no. 3/2006, pp. 149-156, 2006. 


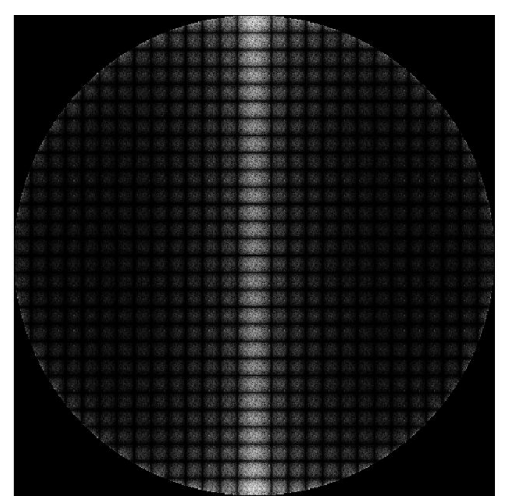

(a) $P=8 \mu m, \Delta=7 \mu m$

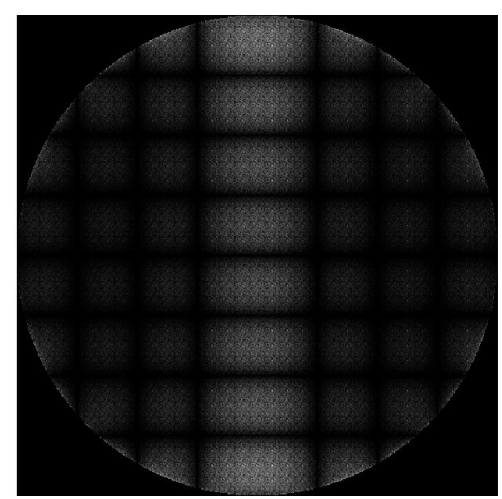

(b) $P=8 \mu m, \Delta=2 \mu m$

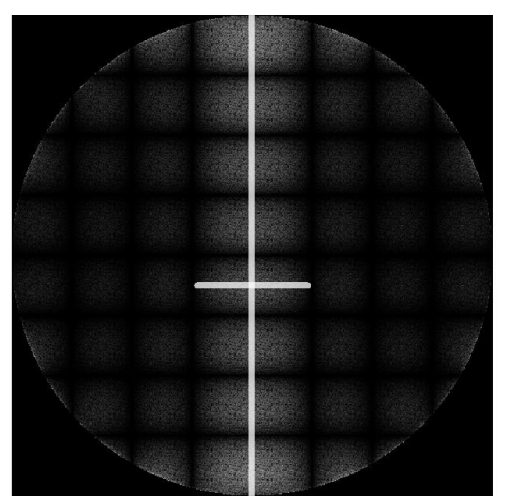

(c) $P=20 \mu m, \Delta=2 \mu m$

Figure 1: The $\left|\mathcal{E}_{z}\left(k_{x}, k_{y}\right)\right|$ of Equation 10 are shown as gray scale images in three cases for a display size of $1080 \times 1920$ pixel. $\hat{E}_{y}[n, m]$ is the same for all three cases and equals to $e^{j \phi[n, m]}$, where $\phi[n, m]$ is generated randomly for each $n$ and $m$ independently from a uniform distribution between 0 and $2 \pi$ with 8-bit precision. In these figures, the frequency axes are along the spatial frequency variables $k_{x}$ and $k_{y}$ that correspond to the horizontal and vertical axes, respectively and $k_{x}, k_{y} \in[-k, k)$. The centers of the images correspond to $\left(k_{x}, k_{y}\right)=(0,0)$ point. The frequency increases along the horizontal axis from left to right, and along the vertical axis from top to bottom. The colormap is in log scale for better illustration. The horizontal and vertical lines in Figure 1c indicate the locations of $1 \mathrm{D}$ cross sections shown in Figure 2.

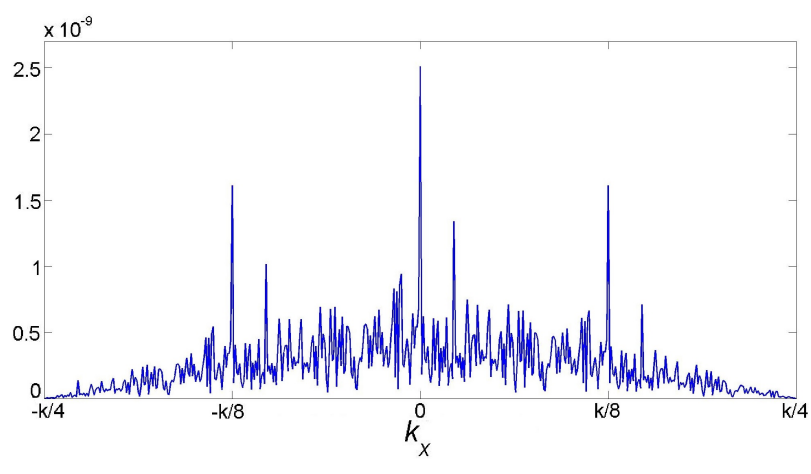

(a) Horizontal profile of Figure $1 \mathrm{c}$ at $k_{y}=k / 8$

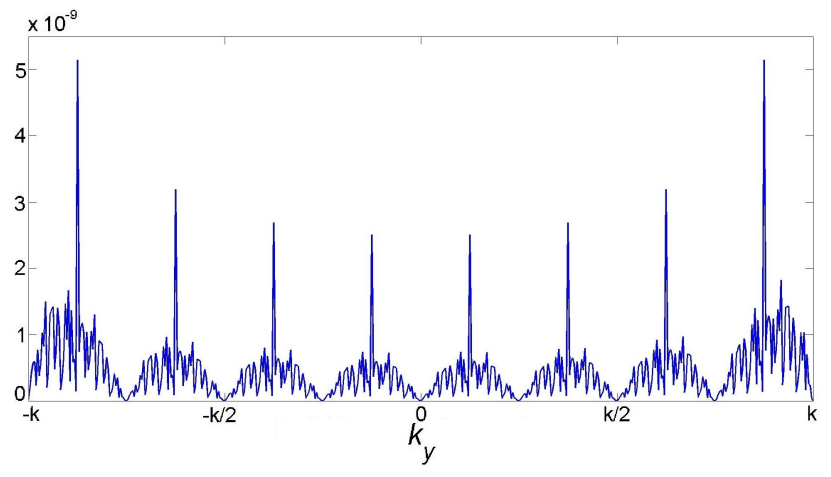

(b) Vertical profile of Figure $1 \mathrm{c}$ at $k_{x}=0$

Figure 2

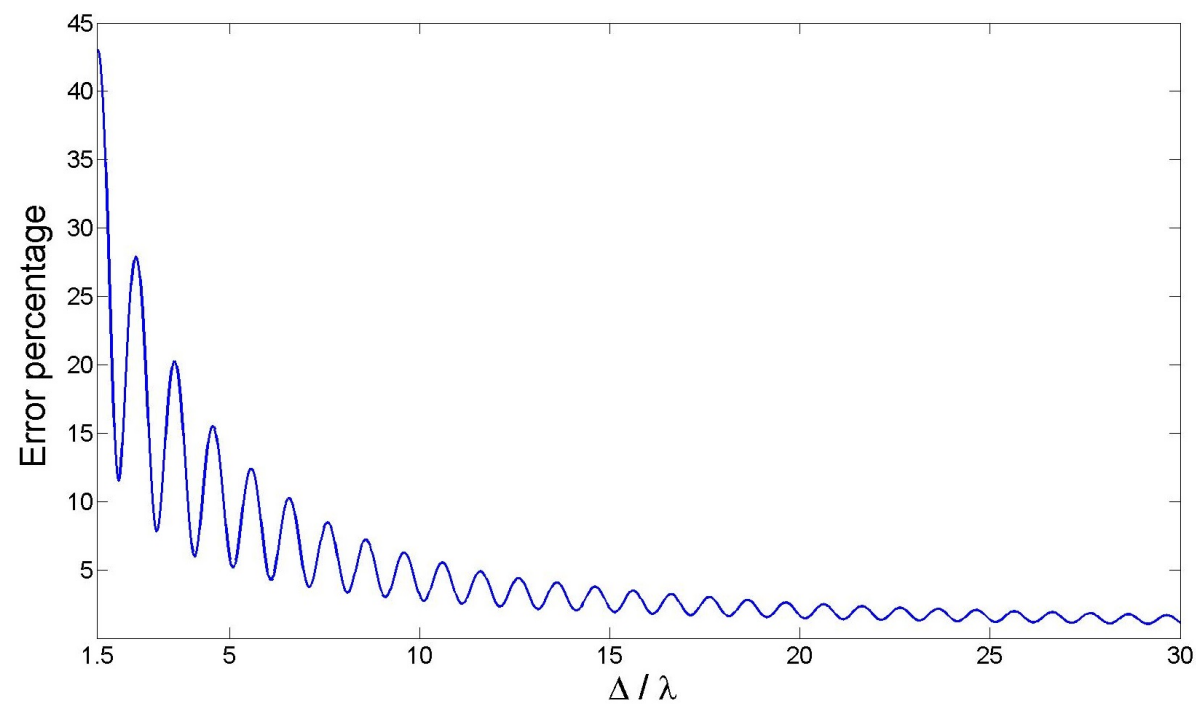

Figure 3: The error in the scalar approximation, $\epsilon$, is shown for a single pixel as a function of the normalized width of the active region with respect to the wavelength, which is assumed as $\lambda=500 \mathrm{~nm}$. 